myocarditis (cardiogenic shock secondary to infective myocarditis that did not respond to medical treatment) received ECMO for 42$171 \mathrm{~h} ; 13$ patients with nonfulminant myocarditis were not given ECMO.

In this study, 10 out of 14 patients with fulminant myocarditis survived the acute phase, equaling the best survival rates of previous studies where ECMO was used mainly as a bridge to transplantation. Levels of creatine phosphokinase MB and blood urea nitrogen were higher in the four patients who died than in those who survived. Over the initial 6-12 months of follow-up, myocardial damage (indicated by fractional shortening) decreased markedly in the fulminant group, and reached levels similar to that seen in the nonfulminant group. No additional patients died or required heart transplantation during $~ 50$ months of follow-up; however, the good results seen in this study might stem from careful initial patient selection.

Limitations of this study include its retrospective single-center design and small size. Nonetheless, the authors conclude that ECMO has significant advantages over alternative circulatory support technologies, in that it is minimally invasive, quick and easy to use, and can result in better survival and fewer complications.

Caroline Barranco

Original article Asaumi Y et al. (2005) Favourable clinical outcome in patients with cardiogenic shock due to fulminant myocarditis supported by percutaneous extracorporeal membrane oxygenation. Eur Heart $J$ [doi:10.1093/eurheartj/ehi411]

\section{Locations of plaque rupture in coronary arteries}

A recent prospective study carried out in Korea and the US investigated the location of plaque ruptures in native coronary arteries with the use of three-vessel intravascular ultrasound (IVUS).

Hong and colleagues performed IVUS on 393 patients with either acute coronary syndrome or stable angina pectoris, and found that 206 patients-158 with acute coronary syndrome and 48 with stable angina pectoris-had ruptured plaques. A total of 273 individual plaque ruptures were detected by IVUS: 143 were located in the left anterior descending artery (LAD), 90 in the right coronary artery (RCA), and 40 in the left circumflex artery (LCX).
Following IVUS imaging, the exact locations of the plaque ruptures were analyzed. It emerged that the vast majority $(83 \%)$ of LAD plaque ruptures were located between 10 and $40 \mathrm{~mm}$ from the LAD ostium; however, LCX plaque ruptures appeared to be evenly distributed throughout the length of the artery, and most RCA plaque ruptures appeared either between 10 to $40 \mathrm{~mm}$ from the ostium (48\%) or further than $70 \mathrm{~mm}$ from the ostium (32\%).

Concluding that plaque ruptures are located predominantly in the proximal region of the $L A D$, the proximal and distal regions of the RCA, and the whole of the LCX, the authors speculate that data from this study may be of use for devising strategies to detect rupture-prone plaque.

Christine Kyme

Original article Hong MK et al. (2005) The site of plaque rupture in native coronary arteries-a three-vessel intravascular ultrasound analysis. J Am Coll Cardiol 46: 261-265

\section{Similar clinical benefits with dual-chamber and single- chamber pacemakers}

Guidelines that recommend dual-chamber pacing over single-chamber pacing for treatment of atrioventricular block have recently been questioned. Previous clinical trials supporting these recommendations were not randomized, with younger patients often selected to receive dual-chamber pacemakers. The United Kingdom Pacing and Cardiovascular Events (UKPACE) trial compared the clinical outcomes of elderly patients treated with either singlechamber ventricular pacing or dual-chamber pacing. A total of 2,021 patients with high-grade atrioventricular block, $\geq 70$ years of age, were randomly assigned to receive a single-chamber ventricular pacemaker (1,009 patients) or a dual-chamber pacemaker (1,012 patients). Mortality rates and other cardiovascular events were recorded during a follow-up period of up to 4.6 years. The mean annual mortality rate was $7.2 \%$ in the single-chamber group and $7.4 \%$ in the dual-chamber group. Reported incidences of secondary outcomes were also similar between the two groups, including atrial fibrillation, heart failure and stroke, transient ischemic attack or other thromboembolism. Procedural complications were, however, more frequent in patients receiving dual-chamber pacemakers 